\title{
Towards realistic core-failure-resilient scheduling and analysis
}

CISTER - Research Center in

Real-Time \& Embedded Computing Systems

\section{(ㄷ) The schedulling problem}

- Goal: Meeting all task deadlines on a multicore platform even when a core suddenly fails and is rendered unusable.

- Model: When a core fails, whichever task was running there is killed but its deadline must still be met .

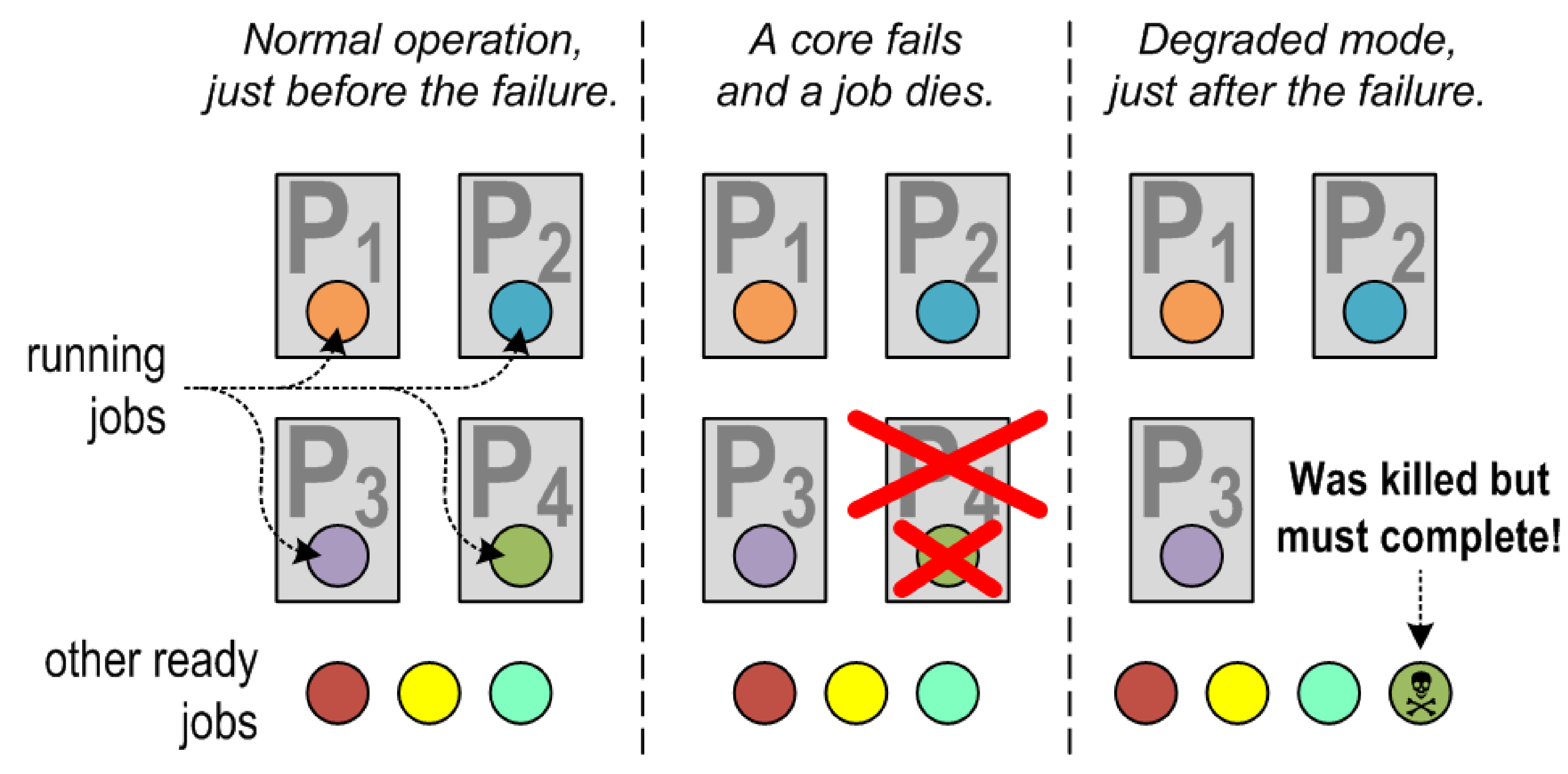

- Our basic scheduling arrangement and analysis for this problem (RTCSA 2015) makes many simplifying assumptions. We want to make it more realistic.

\section{(2) the baselline appproach}

- Global fixed-priority scheduling.

- Generalisation of two simple but "faulty" ideas:

- Full task replication (Resource-inefficient!)

- Restart task upon failure (May be too late!)

- For each job by task $\mathrm{T}_{\mathrm{i}}$, release a copy job after a time offset $\mathrm{O}_{i}$, relative to the main job.

- smaller $\mathrm{O}_{\mathrm{i}}$ : more redundant execution.

- bigger $\mathrm{O}_{\mathrm{i}}$ : harder to meet deadline.

- Optimal $\mathrm{O}_{\mathrm{i}}$ : the biggest value that allows provably meeting deadlines in every case.

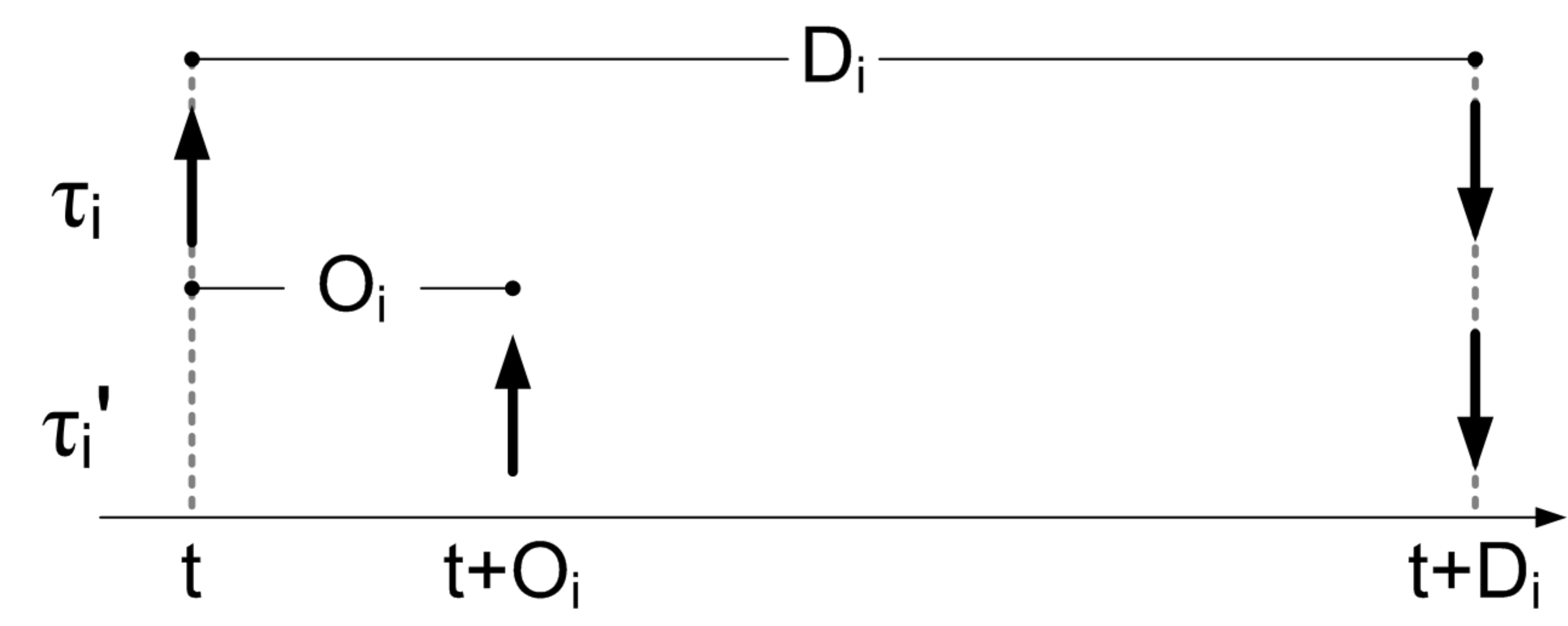

Borislav Nikolić and Konstantinos Bletsas

\{borni, ksbs\}@isep.ipp.pt

\section{(3) Guitical sections}

- Provisions for resource sharing under some adaptation of an existing protocol are needed.

- But what happens if a task dies while executing a critical section?

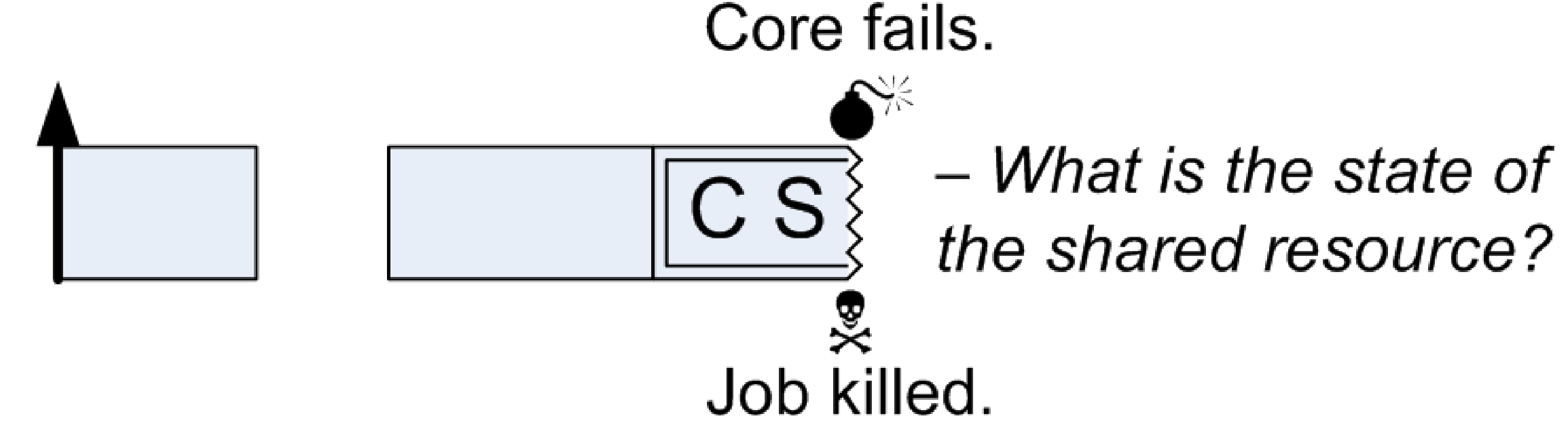

- Transaction semantics (with COMMIT and ROLLBACK) appear as an appropriate solution.

\section{(44) Undifrest resomrae sharting}

- With job copies, all resources suddenly become shared (between the main job and its copy)!

- Possible solutions:

- Critical sections everywhere (inefficient).

- Code-level analysis also considering $\mathrm{O}_{\mathrm{i}}$, in order to rule out some access hazards.

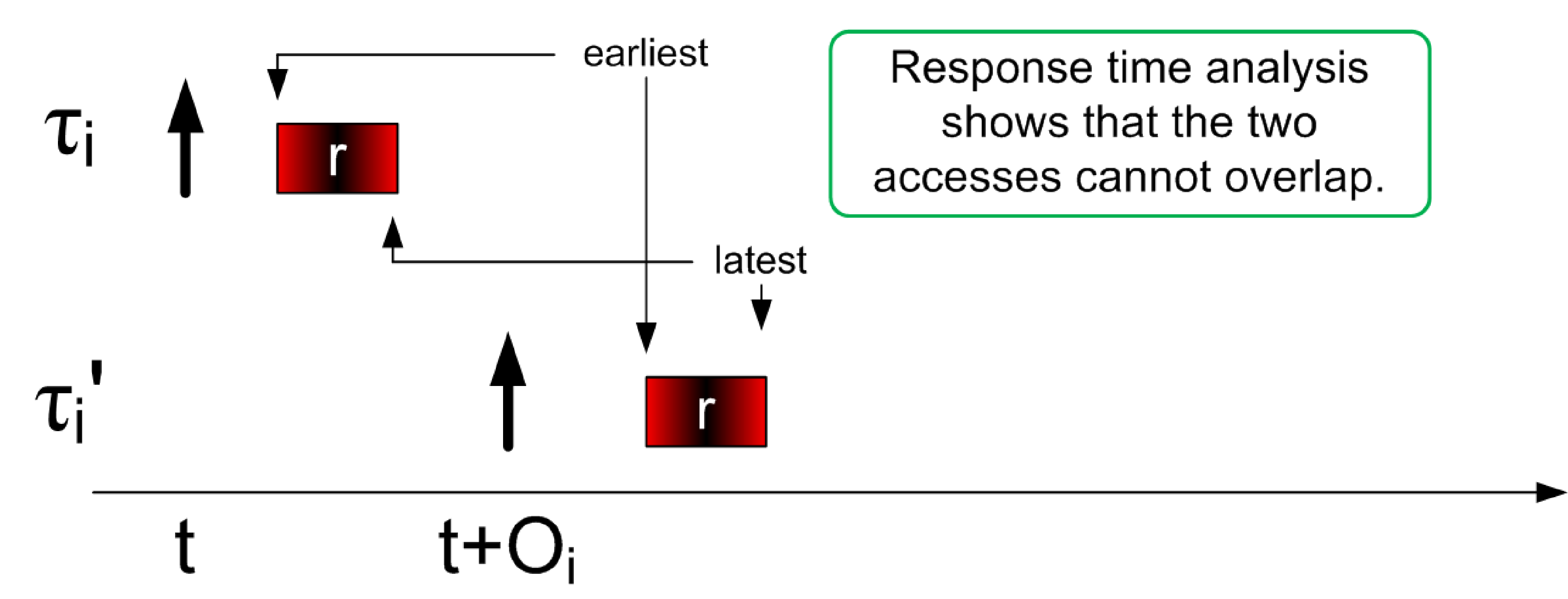

\section{((5)) Implementation aspects}

- Facility for detecting/handling core failures.

- Facility for launching, tracking and terminating jobs early.

- Incorporation of overheads into the analysis, taking into account the actual implementation.

CISTER Research Centre/INESC-TEC ISEP, Polytechnic Institute of Porto Rua Dr. Ant ${ }^{\circ}$ Bernardino de Almeida, 431 4200-072 PORTO Portugal tel: $+351-228340502$ fax: +351-228340509 http://www.cister.isep.ipp.pt cister-info@isep.ipp.pt 